\title{
Strates
}

STRATES Matériaux pour la recherche en sciences sociales

5 | 1990

Conjuguer stratégies et territoires?

\section{À propos des choix résidentiels des périurbains : peut-on parler de stratégies territoriales?}

\section{Martine Berger}

\section{(2) OpenEdition}

1 Journals

Édition électronique

URL : http://journals.openedition.org/strates/1424

DOI : $10.4000 /$ strates. 1424

ISSN : $1777-5442$

Éditeur

Laboratoire Ladyss

Édition imprimée

Date de publication : 31 décembre 1990

ISSN : 0768-8067

Référence électronique

Martine Berger, "À propos des choix résidentiels des périurbains : peut-on parler de stratégies

territoriales? », Strates [En ligne], 5 | 1990, mis en ligne le 16 mars 2007, consulté le 08 septembre

2020. URL : http://journals.openedition.org/strates/1424; DOI : https://doi.org/10.4000/strates.1424

Ce document a été généré automatiquement le 8 septembre 2020

Tous droits réservés 


\title{
À propos des choix résidentiels des périurbains : peut-on parler de stratégies territoriales?
}

\author{
Martine Berger
}

1 Il existe, en matière de choix résidentiels, un champ de possibles entre lesquels les ménages se déterminent : un ménage, ou une personne de ce ménage, choisit de venir s'installer en un lieu, d'y rester, d'en partir; il y a, à un certain moment, arbitrage, donc, en ce sens, stratégie. Empiriquement, on peut saisir des trajectoires résidentielles comme des successions d'états des ménages dans des logements et dégager, à partir de cheminements et de pratiques des types de stratégies, dans la mesure où l'on repère des collections de cas, des régularités de comportements. Si l'on relie ces mouvements des ménages à l'offre de logements, on observe des concurrences entre différents groupes sociodémographiques pour l'accès à certains segments du parc et, en ce sens, enjeux et stratégies. Les apports de l'École de Chicago, qui proposait une image synchronique d'un espace urbain concurrentiel, sont utilement enrichis par des approches diachroniques: migrations intercensitaires, enquêtes mettant en relation cursus résidentiels, professionnels, familiaux.

2 On peut également parler, à propos des ménages périurbains, de stratégies au sens de série d'actions plus ou moins efficaces pour maintenir ou transformer un espace. Espace qui peut être perçu, ou pratiqué, à différentes échelles, par les acteurs-ménages: la commune, le quartier de résidence, le lotissement. On a souvent souligné le degré d'investissement local des propriétaires périurbains, qui constituent la grande majorité des ménages dans les espaces situés à la périphérie des agglomérations. L'espace de résidence joue un rôle très important dans la construction de l'identité sociale, et justifie un investissement qui s'est manifesté tant par le mouvement associatif que par la conquête des mairies, signifiant, avant même l'heure de la décentralisation, une volonté d'intervention sur un espace auquel on entend conserver ou faire acquérir certaines qualités ou certaines valeurs. 
D'où l'opposition entre des stratégies conservatrices de verrouillage de la croissance communale dans le but de maintenir des communautés de pairs, qui sont très souvent le fait des catégories les plus aisées, et des actions tendant à renforcer les équipements municipaux afin de valoriser cet espace de résidence, qui émanent plus souvent des couches moyennes et modestes de la société périurbaine, et supposent la poursuite de la croissance. À une échelle plus fine, l'analyse des pratiques des syndicats de copropriétaires des lotissements, qui tendent à protéger ou promouvoir une certaine image de marque d'un territoire, apporte également des éléments d'information sur les stratégies des différents groupes sociaux.

$4 \quad$ Il s'agit donc bien de stratégies territoriales, et non simplement de stratégies spatiales. L'espace choisi ou défendu est porteur de valeurs, et non simple support de logements d'un certain type, à un certain prix, dans une certaine position. Il a une image de marque, une qualité ; il est susceptible de valorisation ou de dévalorisation. C'est aussi un territoire dans la mesure où le mode d'habiter est un des éléments du positionnement social, de la distinction. Ce besoin de territorialité, qui s'exprime dans l'identification souvent très forte de ses habitants à un espace renvoyant une certaine image de leur position locale, peut paraître paradoxal s'agissant de lieux où, au total, l'on vit peu (la majorité des ménages y sont des couples bi-actifs effectuant souvent de longs trajets quotidiens), mais où la visibilité est très forte, et où l'on restera sans doute longtemps, compte tenu de rôle fixation de l'accession à la propriété : choisie, comme créatrice de patrimoine et d'environnement ? ou subie, du fait de la faiblesse du marché de l'individuel locatif?

La revalorisation du local, du proche, dans ces lieux dont on croit pouvoir contrôler l'évolution, manifeste le déploiement de stratégies territoriales dans ces espaces marqués par une intense mobilité: ampleur des migrations quotidiennes, forte proportion de nouveaux installés dans un parc de logements en cours de constitution, mais aussi départs sanctionnant des échecs ou, au contraire, conduisant à réaliser ailleurs un objectif de promotion. Il serait sans doute intéressant de distinguer à cet égard, sinon deux vagues périurbaines, au moins deux étapes dans le peuplement de ces espaces périphériques de l'agglomération parisienne. Dans un premier temps, l'installation périurbaine ne représentait-elle pas un choix fortement raisonné, un saut, une relocalisation jugée sinon irréversible, au moins de longue durée : d'où une volonté de s'y ancrer pour s'assurer (se rassurer) qu'on a fait le bon choix, et le déploiement de stratégies territoriales d'enracinement. Plus récemment, les flux de mobilité internes aux espaces périurbains l'emportent sur les mouvements d'exurbanisation directe. N'y a-t-il pas, alors, un moindre souci d'identification au territoire ? Le logement y devient une étape dans un cursus résidentiel, un positionnement temporaire, voire un placement que l'on est susceptible de négocier dans un futur proche. Les références spatiales ne deviennent-elles pas moins étroitement locales au fur et à mesure que les espaces périurbains sont en quelque sorte banalisés comme des fragments ou des extensions lâches d'une agglomération ? Le reflux du mouvement associatif ne se limite certes pas aux espaces périurbains, il relève du mouvement social ; mais n'y trouve-t-il pas un facteur d'amplification à la fois dans la banalisation de la mobilité périurbaine et dans le cycle naturel de la propriété et de la vie des ménages (succession de phases d'investissement dans les relations de proximité et de repli sur la famille) ? Il serait intéressant de comparer à cet égard la péri urbanisation et la constitution des 
lotissements pavillonnaires de l'entre-deux-guerres: n'y a-t-il pas eu, là aussi, succession de phases de mobilisation territoriale puis de repli sur la maison?

Il est difficile de faire la part des variations, au cours des deux dernières décennies, des formes de sociabilité, de leur évolution au cours du cycle de vie des ménages, des transformations de la clientèle des accédants à la propriété. Dans quelle mesure l'extension à des couches moins aisées, souvent d'origine plus locale, moins disponibles et moins tentées par la recherche de la distinction dans l'action associative, accentue-telle l'affaiblissement des références territoriales des périurbains? Quel rôle y joue la diminution de la part relative des installations dans des communes rurales par rapport aux programmes réalisés aux marges intermédiaires de l'agglomération, souvent en ville nouvelle? Même si les évolutions récentes banalisent quelque peu l'installation dans le périurbain, diminuant sans doute par là l'intensité des actions d'enracinement territorial, il nous paraît cependant encore tout à fait pertinent d'analyser la mobilité résidentielle périurbaine en termes de stratégies territoriales : il y a bien mise en jeu d'anticipations (dimension projective importante); et, sinon coordination d'acteurs ou de conduites parcellaires, au moins stratégies conservatrices des images des quartiers ou des communes, espaces pensés comme des territoires, c'est-à-dire des lieux sur lesquels on peut agir, l'espace de la vie familiale étant souvent opposé à celui du travail, sur lequel on n'a guère de pouvoir. Ceci d'autant que l'installation périurbaine intervient souvent à une étape importante du cycle de vie (fondation ou agrandissement des familles). Plus encore que la notion de systèmes de pouvoirs s'exerçant sur un espace, et dont les plans d'occupation des sols révèlent les rapports de force, l'analyse de la mobilité résidentielle en termes de collections de stratégies territoriales nous paraît rendre compte des relations des sociétés périurbaines à leurs espaces.

\section{RÉSUMÉS}

Le choix d'un logement par les ménages périurbains d'Ile-de-France peut-il être considéré comme une stratégie territoriale, et non seulement spatiale? Dans la mesure où il s'agit le plus souvent d'accédants à la propriété, leur ancrage local est sans doute plus fort que celui des occupants du parc locatif des banlieues et se manifeste en particulier par leur participation à la vie associative et/ou municipale de leur commune de résidence.

Is the choice of housing place a territorial strategy for households living in the rural-urban fringe?

Do rurban households pursue a territorial - and not only spatial - strategy? It can he assumed that for owner occupiers local attachment may be stronger than that of households living in the rented dwelling stock of suburban areas. It is also supposed that their involvement in local associations and/or institutions is accordingly differing. 
INDEX

Mots-clés : Ile-de-France, Stratégie territoriale, stratégie résidentielle, Choix du logement, Espace périurbain

Keywords : Territorial strategy, residential strategy, Rural-urban fringe

\section{AUTEUR}

MARTINE BERGER

Maître de conférences à l'E.N.S. Fontenay-St-Cloud, elle travaille sur les espaces périurbains en Ile-de-France, en particulier sur les relations entre évolution du parc des logements et division sociale de l'espace. Elle a collaboré au Grand Atlas de la France rurale (Ed. de Monza, 1989) et à l'Atlas démographique et social d'Ile-de-France (DREIF, 1989). 